\title{
Clinical Evaluation of Antihyperglycemic Activity of Metformin in Treatment of Insulin Sensitivity in Polycystic Ovary Syndrome: A double Blind Placebo- Controlled Study
}

Eric Casarini 1*, lan Soyinka2, Clara Vonkeff 3

${ }_{2}^{1}$ DDepartment of Biomedical, Metabolic and Neural Sciences, Phillipines.

Center for Genomic Research, Phillipines

${ }^{3}$ Unit of Endocrinology, Department of Medical Specialties, Philipines.

*Corresponding Author : Eric Casarini , Department of Surgery, Faculty of Medicine, Cairo University, Egypt.Email: Eric@link.com.eg Received date: September 10,2018 ;Accepted date : September 30,2018; Published date: October 09,2018.

Citation for this Article : Eric Casarini, lan Soyinka, Clara Vonkeff Clinical Evaluation of Antihyperglycemic Activity of Metformin in Treatment of Insulin Sensitivity in Polycystic Ovary Syndrome: A double Blind Placebo-Controlled Study. J. Endocrinology and Disorders. Doi: 10.31579/2640-1045/011

Copyright : (C) 2018 Eric Casarini.This is an open-access article distributed under the terms of the Creative Commons Attribution License, which permits unrestricted use, distribution, and reproduction in any medium, provided the original author and source are credited.

\begin{abstract}
Polycystic ovary syndrome is the most common endocrinological disorder affecting 4-12\% of women and also the most controversial. Metformin was logically introduced to establish the extent to which hyperinsulinaemia influences the pathogenesis of the condition. Early studies were very encouraging. Randomized controlled studies and several metaanalyses have changed the picture and put the drug that was once heralded as magic in a much contracted place.

Metformin is one of the longest established oral insulin sensitising agents. For decades its use was restricted to management of type 2 diabetes. However, in the past two decades, its properties as an insulin sensitising agent have been explored in relation to its applicability for women with PCOS. Metformin is an effective ovulation induction agent for non-obese women with PCOS and offers some advantages over other first line treatments for anovulatory infertility such as clomiphene. For clomiphene-resistant women, metformin alone or in combination with clomiphene is an effective next step. Women with PCOS undergoing in vitro fertilisation should be offered metformin to reduce their risk of ovarian hyperstimulation syndrome. Limited evidence suggests that metformin may be a suitable alternative to the oral contraceptive pill (OCP) for treating hyperandrogenic symptoms of PCOS including hirsutism and acne. More research is required to define whether metformin has a role in improving long term health outcomes for women with PCOS, including the prevention of diabetes, cardiovascular disease and endometrial cancer.
\end{abstract}

\section{Keywords}

Estational Diabetes, Insulin-Sensitizing Drugs Infertility, Insulin Resistance, Metformin, Polycystic Ovary Syndrome (PCOS),

Randomized Controlled Trials (Rcts).

\section{Introduction}

Polycystic ovary syndrome (PCOS) is the most common endocrinological disorder affecting $4-12 \%$ of women It has also been the most controversial medical condition and every aspect has received a lot of attention from the nomenclature to the management.

Currently, PCOS refers to a disorder with a combination of reproductive and metabolic characteristics. This has evolved over time with controversy over the definition culminating in the latest consensus which instead of solving the issue created more controversy. In the European Society of Human Reproduction and Embryology/American Society of Reproductive Medicine (ESHRE/ASRM) consensus, at least two of the following features are needed to make the diagnosis; oligo/anovulation, hyperandrogenism, and polycystic features on ultrasound scan [. The Androgen Excess Society, however, recommended that androgen excess should remain a constant feature of PCOS irrespective of the ovulatory status and morphological features of the ovaries For almost three decades, PCOS has been regarded as a life course disease which besides its reproductive features has a long-term impact on the risk of type 2 diabetes mellitus (T2DM) and metabolic syndrome as well as any concomitant cardiovascular disease (CVD) risks.
Metformin is frequently prescribed for type 2 diabetes and has many of advantages including it is weight neutral, and it does not affect the risk of cardiovascular disease ${ }^{2}$ and hypoglycemia Metformin exerts its antihyperglycemic action primarily by inhibiting hepatic gluconeogenesis and by increasing the action of insulin in certain target organs, like muscleand fat Additionally, metformin is progressively used in polycystic ovary syndrome (PCOS) and many studies suggest that metformin could affect pituitary gonadotropin-secreting cells However, the underlying mechanism by which metformin regulates blood glucose levels and/or affects pituitary remains unknown.

Recently, metabolomics has progressed remarkably within the past decade and provided mechanistic insights by correlating biochemical changes with phenotypes. An untargeted metabolomics approach is especially comprehensive in scope and can measure as many metabolites as possible from biological samples simultaneously, without bias. This has great potential for revealing the underlying mechanism of pathophysiology or drug effects. Therefore, these 'metabolomic' studies are seen as a useful tool for the study of metabolic diseases (e.g., diabetes and polycystic ovary syndrome (PCOS)) to investigate systemic alterations in metabolism (e.g., high blood sugar and hormone imbalance) or mechanism of its therapeutic drugs (e.g., metformin). 
It is well known that the pleiotropic actions of metformin are associated with AMP-activated protein kinase (AMPK). The pituitarymediated actions of metformin were also elucidated in PCOS and diabetes. In particular, Lucie Tosca et al. elucidated that metformininduced AMPK activation could exert its action in pituitary cells It was also supported by recent studies that orally-dosed metformin rapidly crosses the blood-brain-barrier $(\mathrm{BBB})$ and accumulates in the pituitary gland and hypothalamus of rats However, the antihyperglycemic mechanism of metformin, associated with the neuroendocrine system, is not fully understood.

These observations suggested that insulin sensitizing agents, such as metformin or troglitazone, should be tested for the treatment of PCOS. These drugs improve insulin sensitivity by different mechanisms, thus determining a subsequent reduction in plasma insulin levels $(8,9)$. Recently, some short-term studies supported this hypothesis, reporting significant reductions of serum androgens in women with PCOS given either metformin or troglitazone. Interestingly, improvements in reproductive abnormalities of these patients have also been reported in some of these studies. On the other hand, other authors failed to observe any clinical or biochemical changes after metformin. These discrepancies are not easily explained. In addition, controlled longterm studies assessing the clinical effects of these treatments are still lacking.

The present study was designed to assess, with a randomized, doubleblind, placebo-controlled protocol, the effects on menstrual abnormalities of a 6-month course of metformin in a group of 23 subjects with PCOS with normal glucose tolerance. Subsequently, 18 of these subjects and 14 additional women with PCOS were included in an open trial to evaluate the long-term effects of metformin on clinical features of the syndrome and to determine any baseline predictors of the treatment's efficacy.

\section{Materials and Methods}

\section{Subjects and study protocols}

Protocol A (double-blind study). Twenty-three caucasian women with PCOS, ages between 18-35 yr and with normal glucose tolerance by the criteria of the World Health Organization (19), were recruited for the double-blind, placebo-controlled study. All these subjects were referred to our division for menstrual abnormalities, with or without hirsutism.

Diagnosis of PCOS was based on the presence of hyperandrogenic chronic anovulation, after exclusion of Cushing's syndrome, late-onset 21-hydroxylase deficiency, thyroid dysfunction, hyperprolactinemia, or androgen secreting tumors, according to recommendations of the NICHHD consensus conference on PCOS (2). No subject had other diseases or was taking medications.

All these women had an abnormal response of serum 17hydroxyprogesterone to GnRH-agonist stimulation (20). Twenty of them had severe oligoamenorrhea (6 or fewer menses per year), whereas 3 women had less severe irregularities in menstrual cycles. In these 3 subjects, anovulation was confirmed by serum progesterone assessment in the luteal phase of the cycle.

The large majority of these subjects were overweight (mean body mass index $30.0 \pm 1.1$, range $\left.19.0-38.7 \mathrm{~kg} / \mathrm{m}^{2}\right)$. Sixteen of them $(70 \%)$ showed increased plasma insulin response to oral glucose, a rough parameter of insulin resistance.

Women were randomly assigned to double-blind oral metformin or placebo for 6 months. The dose of the study drug was increased stepwise, from $500 \mathrm{mg}$ ( 1 capsule) once daily for the first week to 500 $\mathrm{mg}$ bid for the next week, and to $500 \mathrm{mg}$ tid for a further 24 weeks. Patients were instructed not to modify their usual eating habits throughout the study.

Metformin effects on menstrual abnormalities of women with PCOS were evaluated by assessing post-treatment changes in frequency of cycles. Furthermore, changes in several endocrine and metabolic features of the syndrome were also assessed.
Thus, before and at the end of study, the following were carried out:

1. 1. Assessment of menstrual history, with recording of menses in the 6-month periods before the study and during treatment;

2. 2. Physical examination for body weight, waist/hip ratio, hirsutism score, measured by a modification of the Ferriman-Gallwey method (21), and blood pressure, measured by a mercury sphygmomanometer with the subject in the sitting position, after a rest of at least $5 \mathrm{~min}$;

3. 3. Venous blood withdrawal, after overnight fasting, for serum gonadotropins, androgens (total and free testosterone, dehydroepiandrosterone sulfate, androstenedione), 17hydroxyprogesterone, estradiol, sex hormone-binding globulin and lipoproteins (total and HDL cholesterol, triglycerides);

4. 4.GnRH-agonist challenge (20), with measurement of serum 17hydroxyprogesterone, gonadotropins and estradiol, $24 \mathrm{~h}$ after $0.1 \mathrm{mg}$ buserelin sc, as previously described (22);

5. 5. Oral glucose tolerance test, with plasma glucose and insulin measurements on samples obtained every $30 \mathrm{~min}$ for $2 \mathrm{~h}$;

6. 6. Insulin sensitivity assessment by the glucose clamp technique (see below), in 16 out of 23 women participating in the protocol. The remaining subjects did not give their consent to this procedure.

All baseline evaluations were carried out in the early follicular phase of the menstrual cycle, or after at least 3 months of amenorrhea.

Each woman was asked to report any side-effect during the treatment. In addition, safety parameters (hematology, liver and renal function, serum electrolytes and uric acid) were assessed before and at 2-month intervals during the study.

All subjects gave their informed written consent before entering the study, which was conducted in accordance with the Declaration of Helsinki and approved by the institutional ethical committee.

Protocol B (open, long-term study). Eighteen out of the 23 women included in protocol A received metformin in an open design providing treatment for an unscheduled duration after the completion of the doubleblind study, to assess long-term effects of treatment and to determine any predictors of the efficacy of metformin. Fourteen additional, previously untreated, severely oligoamenorrheic women with PCOS were also included in this protocol. Thus, the total number of subjects participating in the open study was 32, 21 overweight and 11 lean. These additional women with PCOS were evaluated at baseline by the procedures indicated in protocol A, except for the glucose clamp. Subjects participating in this protocol also gave their informed consent before entering the study. A placebo group was not included in this long-term protocol, as it was considered unethical after results of the double-blind study. Moreover, a control group is irrelevant to assessment of therapeutic efficacy predictors.

In all patients, metformin dosage was increased stepwise as indicated above, and the following were assessed at 4-month intervals: changes in menstrual history (main outcome), side effects, body weight, hirsutism score, blood pressure, fasting plasma insulin, serum testosterone, gonadotropins, sex hormone-binding globulin, and lipids. GnRH-agonist challenge was repeated after 4-6 months of metformin in 19 subjects.

To assess the effects of treatment on ovulatory function, serum progesterone levels were repeatedly measured in the luteal phases of subjects experiencing regular menses after metformin treatment. Ten women accepted these additional assessments, which were carried out in an overall total of 39 cycles. Ovulation was presumed to have occurred when serum progesterone exceeded $18 \mathrm{nmol} / \mathrm{L}$.

\section{Insulin sensitivity}

Insulin sensitivity was measured by the euglycemic hyperinsulinemic glucose clamp, as previously described (5). Briefly, after overnight fasting, a primed, continuous insulin infusion (Humulin R, Eli Lilly \& Co., Indianapolis, IN) was started; this was maintained for $120 \mathrm{~min}$, at a constant rate of $80 \mathrm{mU} / \mathrm{m}^{2} \times \mathrm{min}$, which makes it possible to reach steadystate plasma insulin levels in the high in vivo range. Euglycemia was maintained throughout the test with a variable infusion of $20 \%$ dextrose, adjusted by monitoring plasma glucose levels in arterialized venous blood, approximately every $10 \mathrm{~min}$. 
We previously found that in nondiabetic hyperandrogenic subjects endogenous glucose production was negligible at this insulin infusion rate (22). Therefore, the amount of glucose infused into each subject may be considered equivalent to the whole body insulin-induced glucose uptake.

\section{Assays}

Plasma insulin was measured by a specific immunoradiometric assay (cross-reactivity with proinsulin $<5 \%$ ), using a kit from Biosource Technologies, Inc. (Fleurus, Belgium). Serum gonadotropins, androgens, progesterone, 17-hydroxyprogesterone, estradiol, sex hormone-binding globulin and lipoproteins were measured by commercial kits, as previously described.

\section{Statistical analysis}

Differences in baseline characteristics between the two groups (metformin and placebo) were analyzed by Student's $t$ test (twotailed). The Mann-Whitney $U$ test and Wilcoxon matched pairs signed rank sum test were used for hirsutism score data.

The difference in frequency of menstruation before and after the trial, a normally distributed variable, was studied by analysis of covariance, so that the effect of intergroup differences in basal body mass index and androgens could be accounted for.

Free testosterone levels and other relevant clinical, endocrine and metabolic features were analyzed by repeated measures analysis of variance. SPSS, Inc. Release 6.0 (SPSS, Inc., Chicago, IL) was used for these analyses.

For the open long-term study, Student's $t$ test was used to analyze differences in main baseline characteristics of responders and nonresponders. Predictors of response to metformin were identified by logistic regression, by the EGRET program (Statistics and Epidemiology Research Corp., EGRET, Seattle, WA.)

\section{Results}

\section{Double-blind, placebo-controlled study}

Clinical and endocrine features.Table 1 shows the main characteristics of women given metformin or placebo, at baseline and after treatment. At baseline, the placebo group had significantly higher body mass index, whereas serum androgens tended to be higher in the metformin group.

\section{Discussion}

Velazquez et al. (10) first reported, in an uncontrolled study, a reduction of serum free testosterone in 29 nondiabetic women with PCOS, mostly overweight, given metformin for 8 weeks. This protocol was designed to evaluate the effects of attenuation of insulin resistance and hyperinsulinemia on the metabolic and endocrine features of PCOS. Unexpectedly, 3 of these subjects became pregnant, and menstrual cycles were normalized in another 7 , who continued the treatment indefinitely. These findings prompted the testing of insulin sensitizing agents, such as metformin or troglitazone, for the treatment of both the metabolic and the endocrine abnormalities of women with PCOS.

\section{Conclusion}

In conclusion, treatment with the insulin sensitizing agent metformin is effective in many women with PCOS, independently of changes in body weight, in attenuating insulin resistance and hyperandrogenemia and in reversing menstrual abnormalities and chronic anovulation. Insulin sensitizing agents may prove an efficacious therapeutic tool in a large subset of subjects with this common disease.

\section{References}

1. Knochenhauer ES, Key TJ, Kahsar-Miller M, et al. Prevalence of the polycystic ovary syndrome in unselected black and white women of the southeastern United States: a prospective study. J Clin Endocrinol Metab 1998; 83:3078.
2. Diamanti-Kandarakis E, Kouli CR, Bergiele AT, et al. A survey of the polycystic ovary syndrome in the Greek island of Lesbos: hormonal and metabolic profile. J Clin Endocrinol Metab 1999; $84: 4006$.

3. Asunción M, Calvo RM, San Millán JL, et al. A prospective study of the prevalence of the polycystic ovary syndrome in unselected Caucasian women from Spain. J Clin Endocrinol Metab 2000; $85: 2434$.

4. Nestler JE. Metformin for the treatment of the polycystic ovary syndrome. N Engl J Med 2008; 358:47.

5. Metformin Therapy for the Management of Infertility in Women with Polycystic Ovary Syndrome: Scientific Impact Paper No. 13. BJOG 2017; 124:e306.

6. Ferrannini E. The target of metformin in type 2 diabetes. $\mathrm{N}$ Engl $\mathrm{J}$ Med 2014; 371:1547.

7. Madiraju AK, Erion DM, Rahimi Y, et al. Metformin suppresses gluconeogenesis by inhibiting mitochondrial glycerophosphate dehydrogenase. Nature 2014; 510:542.

8. Buse JB, DeFronzo RA, Rosenstock J, et al. The Primary GlucoseLowering Effect of Metformin Resides in the Gut, Not the Circulation: Results From Short-term Pharmacokinetic and 12-Week Dose-Ranging Studies. Diabetes Care 2016; 39:198.

9. Niafar M, Hai F, Porhomayon J, Nader ND. The role of metformin on vitamin B12 deficiency: a meta-analysis review. Intern Emerg Med 2015; 10:93.

10. Glueck CJ, Wang P. Metformin before and during pregnancy and lactation in polycystic ovary syndrome. Expert Opin Drug Saf 2007; 6:191.

11. Moghetti P, Castello R, Negri C, et al. Metformin effects on clinical features, endocrine and metabolic profiles, and insulin sensitivity in polycystic ovary syndrome: a randomized, double-blind, placebocontrolled 6-month trial, followed by open, long-term clinical evaluation. J Clin Endocrinol Metab 2000; 85:139.

12. Unlühizarci K, Keleştimur F, Bayram F, et al. The effects of metformin on insulin resistance and ovarian steroidogenesis in women with polycystic ovary syndrome. Clin Endocrinol (Oxf) 1999; 51:231.

13. Yang PK, Hsu CY, Chen MJ, et al. The Efficacy of 24-Month Metformin for Improving Menses, Hormones, and Metabolic Profiles in Polycystic Ovary Syndrome. J Clin Endocrinol Metab 2018; 103:890.

14. Fleming R, Hopkinson ZE, Wallace AM, et al. Ovarian function and metabolic factors in women with oligomenorrhea treated with metformin in a randomized double blind placebo-controlled trial. J Clin Endocrinol Metab 2002; 87:569.

15. Velázquez E, Acosta A, Mendoza SG. Menstrual cyclicity after metformin therapy in polycystic ovary syndrome. Obstet Gynecol 1997; 90:392.

16. Genazzani AD, Battaglia C, Malavasi B, et al. Metformin administration modulates and restores luteinizing hormone spontaneous episodic secretion and ovarian function in nonobese patients with polycystic ovary syndrome. Fertil Steril 2004; 81:114.

17. Eisenhardt S, Schwarzmann N, Henschel V, et al. Early effects of metformin in women with polycystic ovary syndrome: a prospective randomized, double-blind, placebo-controlled trial. J Clin Endocrinol Metab 2006; 91:946.

18. Essah PA, Apridonidze T, Iuorno MJ, Nestler JE. Effects of shortterm and long-term metformin treatment on menstrual cyclicity in women with polycystic ovary syndrome. Fertil Steril 2006; 86:230.

19. Ibáñez L, Valls C, Ferrer A, et al. Sensitization to insulin induces ovulation in nonobese adolescents with anovulatory hyperandrogenism. J Clin Endocrinol Metab 2001; 86:3595.

20. Carmina E, Lobo RA. Does metformin induce ovulation in normoandrogenic anovulatory women? Am J Obstet Gynecol 2004; 191:1580 
21. Tang T, Lord JM, Norman RJ, et al. Insulin-sensitising drugs (metformin, rosiglitazone, pioglitazone, D-chiro-inositol) for women with polycystic ovary syndrome, oligo amenorrhoea and subfertility. Cochrane Database Syst Rev 2012; :CD003053.

22. Morin-Papunen LC, Vauhkonen I, Koivunen RM, et al. Endocrine and metabolic effects of metformin versus ethinyl estradiol-cyproterone acetate in obese women with polycystic ovary syndrome: a randomized study. J Clin Endocrinol Metab 2000; 85:3161.

23. Costello M, Shrestha B, Eden J, et al. Insulin-sensitising drugs versus the combined oral contraceptive pill for hirsutism, acne and risk of diabetes, cardiovascular disease, and endometrial cancer in polycystic ovary syndrome. Cochrane Database Syst Rev 2007; :CD005552.

24. Nestler JE, Stovall D, Akhter N, et al. Strategies for the use of insulin-sensitizing drugs to treat infertility in women with polycystic ovary syndrome. Fertil Steril 2002; 77:209.

25. Tso LO, Costello MF, Albuquerque LE, et al. Metformin treatment before and during IVF or ICSI in women with polycystic ovary syndrome. Cochrane Database Syst Rev 2009; :CD006105.

26. Palomba S, Falbo A, La Sala GB. Effects of metformin in women with polycystic ovary syndrome treated with gonadotrophins for in vitro fertilisation and intracytoplasmic sperm injection cycles: a systematic review and meta-analysis of randomised controlled trials. BJOG 2013; 120:267.

27. Abdalmageed OS, Farghaly TA, Abdelaleem AA, et al. Impact of Metformin on IVF Outcomes in Overweight and Obese Women With Polycystic Ovary Syndrome: A Randomized Double-Blind Controlled Trial. Reprod Sci 2018; :1933719118765985.
28. Palomba S, Falbo A, Carrillo L, et al. Metformin reduces risk of ovarian hyperstimulation syndrome in patients with polycystic ovary syndrome during gonadotropin-stimulated in vitro fertilization cycles: a randomized, controlled trial. Fertil Steril 2011; 96:1384.

29. Al-Inany HG, Youssef MA, Ayeleke RO, et al. Gonadotrophinreleasing hormone antagonists for assisted reproductive technology. Cochrane Database Syst Rev 2016; 4:CD001750.

30. Practice Committee of the American Society for Reproductive Medicine. Electronic address: ASRM@asrm.org, Practice Committee of the American Society for Reproductive Medicine. Role of metformin for ovulation induction in infertile patients with polycystic ovary syndrome (PCOS): a guideline. Fertil Steril 2017; 108:426.

31. Lord JM, Flight IH, Norman RJ. Insulin-sensitising drugs (metformin, troglitazone, rosiglitazone, pioglitazone, D-chiroinositol) for polycystic ovary syndrome. Cochrane Database Syst Rev 2003; :CD003053.

32. Ehrmann DA, Barnes RB, Rosenfield RL, et al. Prevalence of impaired glucose tolerance and diabetes in women with polycystic ovary syndrome. Diabetes Care 1999; 22:141.

33. Sharma ST, Wickham EP 3rd, Nestler JE. Changes in glucose tolerance with metformin treatment in polycystic ovary syndrome: a retrospective analysis. Endocr Pract 2007; 13:373.

34. Ehrmann DA, Cavaghan MK, Imperial J, et al. Effects of metformin on insulin secretion, insulin action, and ovarian steroidogenesis in women with polycystic ovary syndrome. J Clin Endocrinol Metab 1997; 82:524. 